\title{
Research and Analysis of Urban Housing Remodeling for the Elderly under the Endowment Model of Japan
}

\author{
Shanghai original structure Design Consulting Co., Ltd. Shenyang Branch, Shenyang City, Liaoning Province \\ Postcode:11000
}

\section{Introduction}

Since 2000, China has also entered an aging society, and aging population is continuing to mount with China's 60-yearold and older people reached about 180 million, accounting for the total population of 13.76\%. Till "Twelve-Five", our country's first elderly peak has increased to 8.7 million. The increase of China's elderly population during the period from 2011 to the end of 2015 becomes faster and 222 million was added, and the proportion increased to $16 \%$. By 2030 , the elderly in China will increase to around 400 million, with an annual increase of 10 million, and the growth rate will surpass that of Japan and China will become the country with the most serious aging degree across the world. Therefore, this paper provides a series of analysis and reference for the reconstruction of the old-age housing in China by studying Japan's current supporting facilities.

\section{Inner Space}

The inner space is the residence of the old people for a long time, and it is the precondition of modeling for the elderly, which is different from the ordinary residential space. The implementation of the modeling will certainly reduce all kinds of hidden dangers in life. Japanese local designers put more emphasis on more pragmatic function in design, and by summing up the innovative ideas of design, the general design can integrate and centra- lize the interior. The color of room can directly affect people's senses and mood, so the selection and use of light coffee, beige, cyan can make people feel relaxed. Minimize the use of deep colors which give people a sense of depression. The elderly with inconvenient mobility may use assistive tools, so the design will be more effective if it can handle emergencies timely. A perfect using space can be created under a spatially reasonable structure system. When designing, integrate the space of kitchen, dining room and bedroom, to create better conditions for modeling for the elderly.

\subsection{Kitchen washing}

(1) Reset the height of the kitchen counter. The height of normal counters is: height $/ 2+5 \sim 10 \mathrm{~cm}$. Japanese seniors are shorter than average people because of hunchback, so they can sit down and cook, which is worthy of reference. For example, for the elderly in wheelchairs, the work station can be added $18 \mathrm{~cm}$ in accordance with their length of legs.

(2) Sensor faucets can greatly reduce the bending action and save water effectively. Besides, they can accommodate the elderly who easily forget to turn off faucets because of poor memory with convenience, so as to reduce unnecessary potential danger; foam flushing can improve cleaning ability and save water and labor.

\begin{abstract}
With the deepening of Japan's aging and miniaturizing of family structure, coupled with Japan's economic decline, unreasonableness and abnormal supply brought by the old pension system and ancillary equipment will bring a series of problems. Social endow-ment is the trend of the times. In this paper, the author expounds the problems encountered in the reconstruc-tion of urban housing under the old-age model in Japan, and provides a comprehensive ser-vice to meet the needs of the elderly and be in line with their requirements and life style in the light of the housing, supporting space, public access, internal and external environment and public facilities. And the author creates economic and practical, comfortable and convenient, safe and healthy supporting residential facilities in line with living habits of the local elderly, and on this basis, the use of the Internet of things can better support the old-age housing and promote the rapid development of oldage reform.
\end{abstract}

Key words: Japan; aging; endowment; urban housing; reconstruction

Published online: 30th Sept, 2017

(3) The use of dishwasher in the developed countries has reached $70 \%-80 \%$. Japan's electronic and electrical technology is developed and they really focus on and are strict in food hygiene. So dishwashers should not only 
reduce the burden of labor, but also ensure the function of sterilization and disinfection, so as to be healthier.

(4) In the bathroom, we should pay attention to strengthen the stability of the washing table, and ensure that there is enough space below to prevent being stumbled by the edge. The height of the wash basin and cabinet should remain around $65 \mathrm{~cm}$. The mirror height in $80 \mathrm{~cm}$ is suitable; $60 \mathrm{~cm}$ width should be set in front of the makeup mirror to make people can stand or sit to complete the washing; the handrail should be installed in the edge to prevent being stumbled. The contact point between the washbasin and the faucet should be extended down $30 \mathrm{~cm}$, extend-ing to $10 \mathrm{~cm}$ below the faucet outlet.

(5) The bathroom is designed to use Sit Down Shower to prevent you from falling down and being hurt when you step into or climb out of the bathtub. Fog bath steam can increase the temperature in a short period of time, so it can save more water than hip bath and is very suit-able for heart disease patients. Use a thermostat faucet to completely control the water temperature and reduce irrita-tion to the skin.

(6) The use of intelligent toilet cover originated from the early American hospital old-age health care, initially with warm water rinse function, afterwards the automatic drying, washer heating and anti-bacterial deodorant functions are added. Intelligent Sweeper not only facilitates the mobility of the elderly, but also reduces the likelihood of hunchback. Intelli-gent drying racks can reduce mobility problems, convenient life, and make life more convenient.

\subsection{Living and environment}

(1) The bed height should be set at $40 \sim 50 \mathrm{~cm}$. For people in wheel- chairs, the height of the bed should be the same as the wheelchair. In terms of health, the old man is not suitable for sleeping on too soft bed. Handrails can be set at the edge of the bed to facilitate the elderly to get up.

(2) The humidity of the room should be taken seriously, because the old people is more sensitive to the change of humidity and easy to have problems such as pruritus and frowsty bosom. If the humidity remains above 65\%, indoor environment will be humid and muggy, easy to breed mold and mites; below $40 \%$, indoor window will have condensation, people inside will get flu easily, and the skin will be too dry and static will be generated. Humidity control between $50 \%$ to $70 \%$, then it can be supple-mented with humidifier solution, and some decorative materials can also be placed to absorb humidity.

(3) Universal use of floor heating. The elderly are more afraid of the cold, and always feel cold below the knee, so the floor heating is the most comfortable. It can heat from the bottom up to the foot, and is smokeless, tasteless, environmentally friendly, and low-carbon. And once the air is not dry, the damage of the skin can be reduced. Besides, the floor heating can use space effec-tively without any waste.

(4) Electrical switches and sockets. In general, the height of the electric is set to $120 \mathrm{~cm}$, but considering the height of the elderly in Japan, it is reduced to about $90 \mathrm{~cm}$ to avoid be stumbling by unnecessary power cords.

\section{Public facilities and public space}

2.1 The design of public space is a weak link in the development and design, but the usage rate plays an important role in the life of the elderly. Achieve 0 distance from the residential area to the home, and make it convenient and fast to reach the residence. Between the building and the corridor, enough space should be saved as far as possible to meet walking, small activities, and wheelchair rotation for the elderly. The corridor is provided with a continuous handrail to give full play to residential properties.

2.2 The elderly after the age of 65 are easily to be completely bedridden and have complication because of careless fall. Pay attention to the safety of walking, and the handrail should be set at the corridor to make standing up easier. $35 \mathrm{~cm}$ is the most comfortable diameter for handrail.

2.3 Set the barrier-free ground, remove the threshold, and choose to use the sliding doors with lifting rails. For floor, the selected material should not only be antiabrasive, antimildew, antiskid, environmentally friendly and soft, but also meet all warming demands and have sound-proof function. In terms of elevator use, barrier-free facilities should be installed in the elevator, such as safe handrails with the emergency alarm system.

\section{Conclusion}

Through a series of elaboration, this article shows that: with the progress of the times, products that are more consistent with ergonomics and overall research are designed for the elderly, taking psychological, physiological and behavioral features as the starting points. Achieving the improvement of modeling for the elderly has farreaching significance and long-term meaning to keep up with the times and sustainable development. From the perspective of architecture, this is an effective solution to the crisis of population aging, and it also creates good foresight and timeliness for coping with the design for elderly in our country. 